\title{
Evaluation of GMDH and MLP Networks for Prediction of Compressive Strength and Workability of Concrete
}

\author{
Niloufar GHAZANFARI ${ }^{1}$, Shayan GHOLAMI ${ }^{2}$, Alireza EMAD ${ }^{3}$, Mohammad SHEKARCHI $^{4}$ \\ M.Sc. Student, Department of Civil Engineering, University of Tehran, Tehran, Iran \\ B.Sc. Student, Department of Civil Engineering, University of Tehran, Tehran, Iran \\ B.Sc. student, Construction Material Institute (CMI), University of Tehran, Iran \\ Professor, Department of Civil Engineering, University of Tehran, Tehran, Iran \\ *Corresponding author: N.ghazanfari@ut.ac.ir
}

\begin{abstract}
Measurement of compressive strength and workability of concrete as the most important mechanical properties of concrete have always been a significant topic to designers. Considering a large number of parameters influencing the mixed design of concrete and the reduction in the number of test samples, today various inferential algorithms are used for discovering relationships, predicting mechanical properties, and identifying nonlinear patterns along with optimizing concrete mixtures. Among these algorithms, artificial neural networks are highly effective and reliable alternative for complicated systems with uncertain structure in which the aim is to obtain different kinds of relationship between the input and output variables with high orders. The methodology used in this study is based on MLP and GMDH artificial neural networks, which lack theoretical basis and are known as inverse engineering (IE) or black box models. To present a model for prediction of these parameters, two artificial neural network approaches were investigated and the performance of GMDH model, when comparing the laboratory results with the predicted values, suggests acceptable accuracy of this model in evaluation of compressive strength and slump.
\end{abstract}

Keywords: concrete compressive strength, workability, Artificial Neural Network (ANN), Multi-Layer Perceptron (MLP), Group Method of Data Handling (GMDH)

\section{Introduction}

Compressive strength and workability of concrete are the most important mechanical properties of concrete which are considered as the main criteria in determining the properties of desirable structural grade concrete. Compressive strength refers to the level of uniaxial compressive stress when the element becomes fully dissociated and other concrete properties such as elastic modulus and water tightness appear to have direct relationships with the compressive strength. Hence, the compressive strength is commonly used as the main criterion in defining the required quality of concrete, and an accurate estimation of the strength before the placement is a practical need of construction engineers. 
On the other hand, the workability, which according to ACI definition refers to the ease with which concrete can be mixed, placed, consolidated and finished. A mix that is difficult to place and consolidate will increase the cost of handling, and lead to poor strength, durability and appearance. This property of concrete is generally known to affect the consistency, flow ability, pump ability, compatibility, and harshness of a concrete mix. Thus, concrete workability is a very crucial factor that must be considered in order to produce high quality concrete.

Due to the importance of the research topic, various studies have been conducted in this regard and different traditional statistical models and machine learning methods have been presented so far. Linear and nonlinear multiple regression are the most common approaches for estimating the behavior of concrete. However, in recent years the use of computer-aided modeling for predicting the mechanical properties of construction materials is growing. Since the relationship between components and concrete properties is highly non-linear, mathematically modeling the compressive strength of concrete based on available data is difficult. Several methods have been employed using artificial intelligence (AI) to estimate these properties and solve the problem of interest. Among these methods artificial neural networks (ANN) is one of the most powerful tools for modeling complex processes and can capture complex interactions among input and output variables in a system without any prior knowledge about the nature of these interactions.

The concept of ANN, which is, in essence, an attempt to imitate the human brain's learning capabilities, was first presented by McCulloch and Pitts [1] in 1943 by investigating networks of interconnected neurons, with each neuron treated as a simple binary logic computing element. As the basic concepts propounded in the issues related to it enjoyed a solid mathematical basis, over the last decades there has been an increasing interest in using machine learning methods combined with neural network techniques to create autonomous systems that can be used to make prediction for a specific application, such as pattern recognition or data classification.

Artificial neural networks owe their development to the attempts of Haykin [2] in the past two decades. Welstead [3] used back-propagation algorithm (BP), which utilizes the gradient-descent method to minimize the error function and represent complicated associations between patterns.

Kasperkiewicz et al [4] employed fuzzy-based neural networks method (fuzzy-ARTMAP) to estimate the compressive strength of HPC concrete, and obtained the optimal mixed design. Yeh [5] used modified ANN model and established a new approach. By presenting "Response Trace Plots", he also estimated the slump of concrete containing slag and fly ash (FSC). Bai [6] managed to estimate the workability of concrete with metakaolin and fly ash, using the three parameters of slump, compacting factor, and Vebe time. Later other researchers including Oztas et al [7], Yeh [8, 9], Chine et al [10], and Bilgil [11] presented some models for prediction of concrete workability using artificial neural network and regression analysis. Regarding the obtained data their suggested ANN models were more precise and accurate in comparison with other existing regression approaches. Ramezinianpour et al [12] used multilayer perceptron neural networks (MLP) based on the back-propagation paradigm (BP) and presented a network with two internal hidden layers to estimate the strength properties of concrete with the rice husk ash, metakaolin, silica fume, and limestone powder. 
Based on previous studies, AI-based methods, that lack theoretical basis and are known as inverse engineering or black box models, have proven to be more superior than the previous conventional statistical modeling [13-16]. The method used in this study is based on two neural network approaches: multilayer perceptron (MLP) and group method of data handling (GMDH).

In this study, we aimed to assess and compare the prediction accuracy of these two methods in modeling concrete slump flow and compressive strength of concrete incorporating slag, fly ash, and super plasticizer.

\section{Materials and methods}

The basic structure of artificial neural networks (ANN) is simulation of the complex function of brain cells with a large number of interconnected units working in parallel. ANN consists of multiple layers of simple processing elements called as neurons or nodes. Based on the direction of information propagation in networks, ANNs can be classified into two major types, feedforward and recurrent networks. In feedforward networks, the information moves in one direction only from the input nodes to the output nodes through network connections. Unlike feedforward networks, the connection between the units in recurrent networks form a cycle.

Fig.1 represents application of feed forward networks for modeling processes. Patterns are presented to the network via the input layer, which communicates to one or more hidden layers where the actual processing is done via a system of weighted connections.

Many kinds of neural networks have been explained and demonstrated based on learning algorithm. One of the most commonly used feedforward ANNs in function approximation is Multilayer perceptron (MLP) due to their simplicity in implementation and quite satisfying results they give.

Group Method of Data Handling (GMDH) is another neural network modeling algorithm which is known also as Polynomial Neural Networks. The GMDH algorithm is based on an inductive self-organizing approach to the estimation of black box models with unknown relationships between variables (Vissikirsky and Stepashko, 2005).

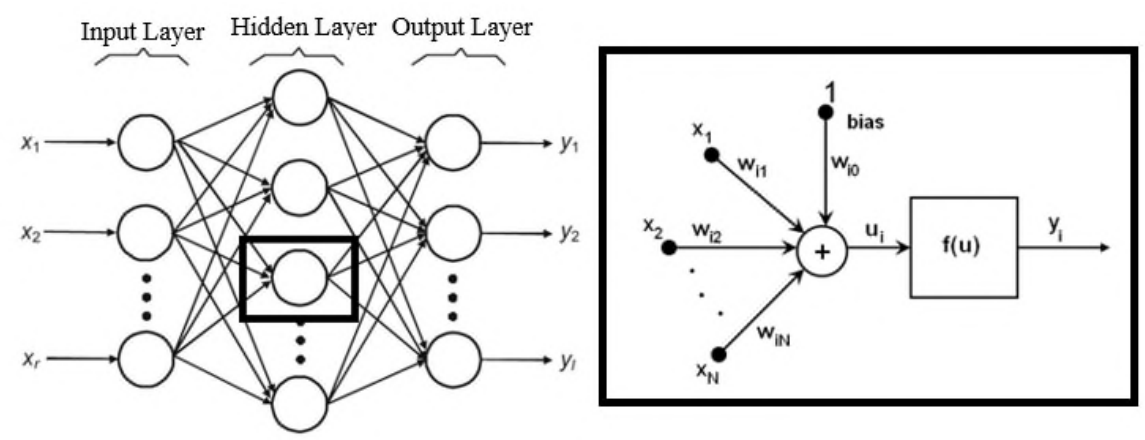

Fig. 1: Feed-forward neural network 


\subsection{MLP neural network}

The multilayer perceptrons (MLP) is a central model of neural network, which frequently used to extract patterns and detect trends of a great variety of real-world problems. To overcome the limitation of single perceptron feed forward neural networks, MLP networks have been introduced. However, too many hidden layers can degrade the network performance. The basic concept of a single perceptron was introduced by Rosenblatt in 1958. Such a network consists of several perceptron layers, where the outputs of the perceptrons of each layer are connected to the inputs of the perceptrons of the next layer. The net input signal is then subjected to an activation function $g$ to produce an output signal $y$. Two of the most used activation functions are the sigmoid function, $\mathrm{g}(\mathrm{u})=\tanh (\mathrm{u})$, and the linear function, $\mathrm{g}(\mathrm{u})=\mathrm{u}$.

The fundamental principle of training process of the MLP is known as the error-backpropagation algorithm, consisting of two phases: feedforward and error-backpropagation. Usually a gradient descent algorithm is used to adapt the weights based on a comparison between the desired and actual network response to a given input stimulus.

In the feed-forward phase, one sample with one or more inputs, depending on the topology of the network, is introduced in the input layer and the outputs of the network are calculated by forwarding the outputs of the neurons on each layer as inputs to the neurons on the following layer, down to the output layer. The error BP phase is performed by first calculating the square error between the desired output $T=\left(t_{1}, t_{2}, \ldots, t_{p}\right)$ and actual output of neurons $o u t=\left(o u t_{1}, o u t_{2}, \ldots, o u t_{p}\right)$, as given by Eq (1):

If total number of algorithms used in training process of network is $\mathrm{Q}$ and the output layer has $\mathrm{P}$ neurons, the error function that is minimized, can be expressed on the form Eq (2):

$$
\begin{aligned}
& \operatorname{Er}(q)=\sum_{k=1}^{p}\left(t_{k}(q)-\text { out }_{k}(q)\right)^{2} \\
& { }^{(1)} \operatorname{Er}=\frac{1}{Q} \sum_{q=1}^{Q} \sum_{k=1}^{p}\left(t_{k}(q)-\text { out }_{k}(q)\right)^{2}, \quad q=1, \ldots, Q
\end{aligned}
$$

Starting from the output layer and going backward in the direction of the first layer, the weights are adapted by delta rule, presented in Eq (3).

$$
\nabla_{k j}=-\frac{\partial E r(q)}{\partial w_{k j}} \eta
$$

Where a constant coefficient is $\eta$ determines the learning rate, and $\nabla_{k j}$ is learning gradient of error related to the connection between neuron $\mathrm{j}$ in hidden layer and neuron $\mathrm{k}$. 


\subsection{GMDH neural network}

Group Method of Data Handling (GMDH) algorithm is a combination of mathematical modeling approaches and black box nonlinear system identification concept, which was first proposed by Ivakhnenko in 1976. He made the neuron a more complex unit featuring a polynomial transfer function. The interconnections between layers of neurons were simplified, and an automatic algorithm for structure design and weight adjustment was developed.

This method can be assumed as a specific type of supervised Artificial Neural Networks (ANN) which uses the idea of Natural selection to control the size, complexity and accuracy of network. The main application of GMDH is modeling of complex systems, function approximation, nonliniear regression, and pattern recognition. [17]

The volterra- Kolmogorov-Gabor (VKG) polynomial function is used as the transfer function to create the initial model. The Kolmogorov-Gabor polynomial function is expressed by:

$y=a_{0}+\sum_{i=1}^{m} a_{i} x_{i}+\sum_{i=1}^{m} \sum_{j=1}^{m} a_{i j} x_{i} x_{j}+\sum_{i=1}^{m} \sum_{j=1}^{m} \sum_{k=1}^{m} a_{i j k} x_{i} x_{j} x_{k}+\ldots$

Where, $X=\left(x_{1}, x_{2}, \ldots, x_{m}\right)$ is the vector of input variables, $y$ is output variables and $A=\left(a_{1}, a_{2}, \ldots, a_{m}\right)$ is the vector of the coefficients or weights in each neuron, and $a_{0}$ represent the bias component in this algorithm. At the first layer of network, an algorithm, using all possible combinations by two from inputs variables, generates the first population of Partial Descriptions (PD). Total number of neurons created on the first layer is defined by the combination of the two input variables $l=\left(\begin{array}{l}\mathrm{m} \\ 2\end{array}\right)$. Layers are grown based on error criteria at each level. Performance is evaluated at each level of the generation of the layers. Next layer starts with maximum possible number of neurons, and determining weights, and then frozen. [18,19] This is different from the BP technique where all of the layers may participate simultaneously in the training process. The training of GMDH network in the $S_{\text {th }}$ iteration to form second order polynomials is formulated as follows:

$y=h\left(w, X^{(s)}\right), s=1, \ldots, m$

Where, $w$ is the vector of coefficients in each neuron, $X$ represents the vector of the network input parameters, and $m$ denotes the number of iterations of the network training process. The sample dataset can be divided to two subsets, the training dataset and the testing data set.

In this approach, one parameter called "Selection Pressure" must be set as proper threshold to determine the number of neurons in each layer. After calculating the coefficients for all the neurons, those which produce the poorest results according to a previously chosen selection criterion must be removed from the layer. Threshold is set to root mean square error (RMSE). Selection values $(0 \leq \alpha \leq 1)$ can vary between zero (no pressure) and one (the maximum pressure of selection). All of the neurons have had a similar structure, where using the external 
Bulletin de la Société Royale des Sciences de Liège, Vol. 86, special edition, 2017, p. 855-868

criterion, the best neurons are classified and proceed to the next stage. The use of critical threshold can have an impact on the accuracy of the resulting values.

$\left\{\begin{array}{c}e_{e}=\alpha e_{\min }+(1-\alpha) e_{\max } \\ e \leq e_{e} \text { (selectionthreshold) }\end{array}\right.$

It should be noted that only one neuron is selected in the last layer.

In this study, the forecasting ability of two neural networks is assessed using correlation factor (R), Mean Square Error (MSE) and Root Mean Square Error (RMSE).

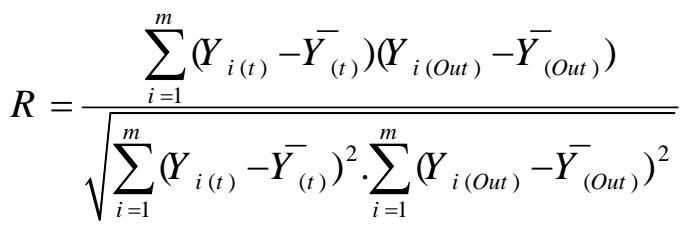

$$
\begin{aligned}
& M S E=\frac{\sum_{i=1}^{m}\left(Y_{i(\text { out })}-Y_{i(t)}\right)^{2}}{m} \\
& R M S E=\sqrt{M S E}
\end{aligned}
$$

Where, $Y_{i(t)}, Y_{i(\text { out })}$ represent the target values and the output values of the network, respectively while $\bar{Y}_{i(t)}, \bar{Y}_{i(\text { out })}$ denote their mean value in $m$ samples.

The architecture of MLP and GMDH networks has been compared schematically in Fig. 2. As can be observed, the mostly used style applies two-combinations of connections. Despite the resemblance to MLP, the structure in the GMDH network is not fully connected.
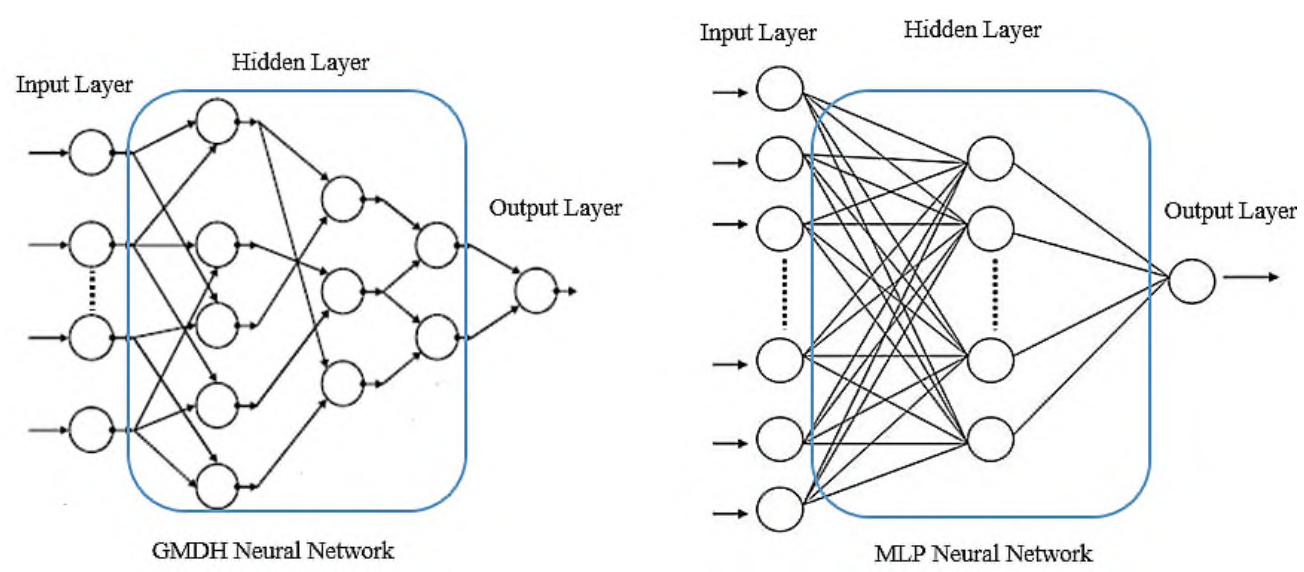

Fig. 2: The schematic architecture of MLP and GMDH neural networks 


\section{Numerical Simulations}

In this part, two simulations based on MLP and GMDH Neural networks are performed in order to predict the compressive strength $\left(\mathrm{F}_{\mathrm{c}}\right)$ and slump $(\mathrm{S})$ of 25 concrete mixtures. Experimental results of Yeh [8] have been used.

The network's input parameters influencing determination of these two factors can be stated by the following function:

$$
(S, F c)=f(C, F A, S l, w, S P, G, F, w / b)
$$

Where, the eight inputs of the network are the values of Cement (C), fly ash (FA), Slag (S1), Water (w), Superplasticizer (SP), Coarse aggregate (G), Fine aggregate (F), and the water.binder ratio(w.b). Materials used in the experiments consisted of type I portland cement, coarse aggregate with a maximum size of $12.5 \mathrm{~mm}$, fine aggregate with a fineness of 2.95, G-type superplasticizer, and cementitious materials of class F fly ash. The SP admixture was HICON HPC 100, conforming to ASTM C-494-Type-G (density: $1.1 \mathrm{~kg} / \mathrm{l}$ ). Specific gravity of each component have been provided in Table 1 .

Table 1: The range of changes and the specific weight of the components of the plan

\begin{tabular}{cccc}
\hline & \multicolumn{2}{c}{ The range of components $\left(\mathbf{K g} / \mathbf{m}^{\mathbf{3}}\right)$} & \\
\cline { 2 - 3 } Component & Minimum & Maximum & Specific gravity \\
\hline Cement & 140 & 350 & 15.3 \\
\hline Fly ash & 0 & 200 & 22.2 \\
\hline Slag & 0 & 240 & 85.2 \\
\hline Water & 150 & 250 & 00.1 \\
\hline SP & 3 & 15 & 20.1 \\
\hline Coarse aggregate & 780 & 1050 & 54.2 \\
\hline Fine aggregate & 640 & 900 & 66.2 \\
\hline
\end{tabular}

At least three cylindrical specimens were prepared from 25 concrete mixes of various proportions of ingredients and tested for compressive strength 28 days. One slump test was also performed for each mix. Measurements from the laboratory are shown in table 2. 
Bulletin de la Société Royale des Sciences de Liège, Vol. 86, special edition, 2017, p. 855-868

Table 2: The details of the mix designs

\begin{tabular}{|c|c|c|c|c|c|c|c|c|c|c|}
\hline \multirow[b]{2}{*}{$\begin{array}{l}\text { Mix } \\
\text { No. }\end{array}$} & \multirow[b]{2}{*}{$\begin{array}{c}\text { Cement } \\
\text { (C) }\end{array}$} & \multicolumn{7}{|c|}{ Component Content $\left(\mathrm{kg} / \mathrm{m}^{3}\right)$} & \multirow[b]{2}{*}{$\begin{array}{l}(\mathbf{C m}) \\
\text { Slump } \\
(\mathbf{S})\end{array}$} & \multirow[b]{2}{*}{$\begin{array}{l}\text { (MPa) } \\
\text { Compressive } \\
\text { strength } \\
\text { (Fc) }\end{array}$} \\
\hline & & $\begin{array}{c}\text { Fly ash } \\
\text { (FA) }\end{array}$ & $\begin{array}{l}\text { Slag } \\
\text { (SI) }\end{array}$ & $\begin{array}{l}\text { Water } \\
(\mathbf{w})\end{array}$ & $\begin{array}{l}\text { Super- } \\
\text { plasticizer } \\
\text { (SP) }\end{array}$ & $\begin{array}{c}\text { Coarse } \\
\text { aggregate } \\
\text { (G) }\end{array}$ & $\begin{array}{c}\text { Fine } \\
\text { aggregate } \\
\text { (F) }\end{array}$ & $\begin{array}{c}\text { Water } \\
\text { binder } \\
\text { ratio } \\
(\mathbf{w} / \mathbf{b})\end{array}$ & & \\
\hline 1 & 147.8 & 2.11 & 178 & 231.3 & 4.4 & 1007.8 & 675.3 & 0.699 & 5 & 25 \\
\hline 2 & 150.2 & 24.1 & 172.8 & 234.9 & 4.5 & 993.1 & 668.2 & 0.69 & 10 & 25 \\
\hline 3 & 151 & 55.3 & 185.3 & 235.1 & 5.1 & 957.9 & 653.7 & 0.613 & 15 & 25 \\
\hline 4 & 150.3 & 74.8 & 194 & 234.9 & 5.5 & 842 & 743.6 & 0.574 & 20 & 25 \\
\hline 5 & 150 & 110.5 & 207.1 & 233.6 & 6.1 & 874.1 & 657.5 & 0.513 & 25 & 25 \\
\hline 6 & 159.3 & 4.1 & 238.4 & 236.4 & 5.2 & 952.4 & 663.5 & 0.606 & 5 & 32.5 \\
\hline 7 & 159.3 & 1 & 238.3 & 236.5 & 5.2 & 968.7 & 646.9 & 0.606 & 10 & 32.5 \\
\hline 8 & 158.3 & 17.3 & 238.9 & 236.4 & 5.4 & 835.7 & 766.6 & 0.583 & 15 & 32.5 \\
\hline 9 & 160.1 & 52.8 & 240 & 235.1 & 5.9 & 914.9 & 640.9 & 0.532 & 20 & 32.5 \\
\hline 10 & 168.3 & 82.2 & 238.3 & 234.6 & 6.4 & 812.3 & 708.2 & 0.493 & 25 & 32.5 \\
\hline 11 & 140 & 82 & 223.4 & 168.6 & 5.8 & 844.7 & 889.1 & 0.391 & 5 & 40 \\
\hline 12 & 140.6 & 66.6 & 239.7 & 171.8 & 5.8 & 832.7 & 896 & 0.398 & 10 & 40 \\
\hline 13 & 162.4 & 64.4 & 239.1 & 180.2 & 6.1 & 812.6 & 879 & 0.4 & 15 & 40 \\
\hline 14 & 184.2 & 66.2 & 239.7 & 190.1 & 6.4 & 789.4 & 854.8 & 0.401 & 20 & 40 \\
\hline 15 & 214 & 66.1 & 239.9 & 234.5 & 6.8 & 842 & 655.5 & 0.464 & 25 & 40 \\
\hline 16 & 156.4 & 77.6 & 239.7 & 168.6 & 6.2 & 823.4 & 886.9 & 0.369 & 5 & 47.5 \\
\hline 17 & 173.9 & 49.2 & 239.2 & 170.8 & 6 & 837.6 & 886.2 & 0.382 & 10 & 47.5 \\
\hline 18 & 194.8 & 48.8 & 239.6 & 179.8 & 6.3 & 814.7 & 868 & 0.385 & 15 & 47.5 \\
\hline 19 & 228.3 & 57.2 & 239.4 & 192.1 & 6.8 & 782.4 & 829.8 & 0.379 & 20 & 47.5 \\
\hline 20 & 293.3 & 36.7 & 239.4 & 203.2 & 7.4 & 882.4 & 664.1 & 0.37 & 25 & 47.5 \\
\hline 21 & 216.3 & 62.3 & 239.7 & 169.1 & 6.7 & 806.5 & 869.8 & 0.339 & 5 & 55 \\
\hline 22 & 243.5 & 32.8 & 239.5 & 173 & 6.7 & 808.2 & 870 & 0.348 & 10 & 55 \\
\hline 23 & 287.5 & 21.2 & 239.9 & 182 & 7.2 & 806.2 & 823.7 & 0.345 & 15 & 55 \\
\hline 24 & 329.6 & 14.5 & 239.9 & 186.4 & 11.5 & 810.1 & 770.8 & 0.339 & 20 & 55 \\
\hline 25 & 352.3 & 0 & 230.9 & 190 & 13 & 806 & 768.7 & 0.348 & 25 & 55 \\
\hline
\end{tabular}

The laboratory results covered five different levels of the required average compressive strengths, about 25, 32.5, 40, 47.5, and $55 \mathrm{MPa}$, and five different levels of workability, about 5, $10,15,20$, and $25 \mathrm{~cm}$ in slump. Therefore, there were $5 \times 5=25$ concrete mixtures.

\section{Results and discussions}

Determination optimum structure of network with proper number of neurons and hidden layers, is one of the most important tasks in MLP networks. The training process is used to determine proper interconnection weights, and desirable results. If an inadequate number of neurons are used, the network will be unable to model complex data, and the resulting fit will be poor. In contrast, using too many neurons in the hidden layers might cause an overfitting or overtraining problem which leads to poor generalization ability on testing dataset. 
In this program, the MLP is feed forward fully connected network, with two hidden layers along with levenberg-Marquadt training algorithm. Two most commonly nonlinear and linear transfer functions, tonsig and pureline, have been used in first and second hidden layers, respectively. It can approximate any function with a finite number of discontinuities arbitrarily well, given sufficient neurons in the hidden layer. After a number of trial, the architecture of the MLP network for prediction the slump and compressive strength of samples, was determined and parameters minimizing the RMSE of each subset were described in the table (3) and table (4), respectively. The results of all the data have also depicted in the figure below.

Table 3: The results of neural network (MLP) associated with slump data

\begin{tabular}{|c|c|c|c|c|c|}
\hline & \multicolumn{3}{|c|}{ Input Data (Slump) } & \multirow[b]{2}{*}{$\begin{array}{c}\text { Number of } \\
\text { neurons in the } \\
\text { first hidden } \\
\text { Layer }\end{array}$} & \multirow[b]{2}{*}{$\begin{array}{l}\text { Number of } \\
\text { neurons in the } \\
\text { second hidden } \\
\text { Layer }\end{array}$} \\
\hline & $\begin{array}{c}\text { Training } \\
\text { Data }\end{array}$ & $\begin{array}{c}\text { Validation } \\
\text { Data }\end{array}$ & $\begin{array}{l}\text { Testing } \\
\text { Data }\end{array}$ & & \\
\hline Contribution (\%) & $\% 66$ & $\% 23$ & $\% 11$ & \multirow{4}{*}{6} & \multirow{4}{*}{4} \\
\hline MSE & 1.74 & 11.35 & 14.44 & & \\
\hline RMSE & 1.32 & 3.37 & 3.80 & & \\
\hline $\mathbf{R}$ & 0.99 & 0.90 & 0.89 & & \\
\hline
\end{tabular}
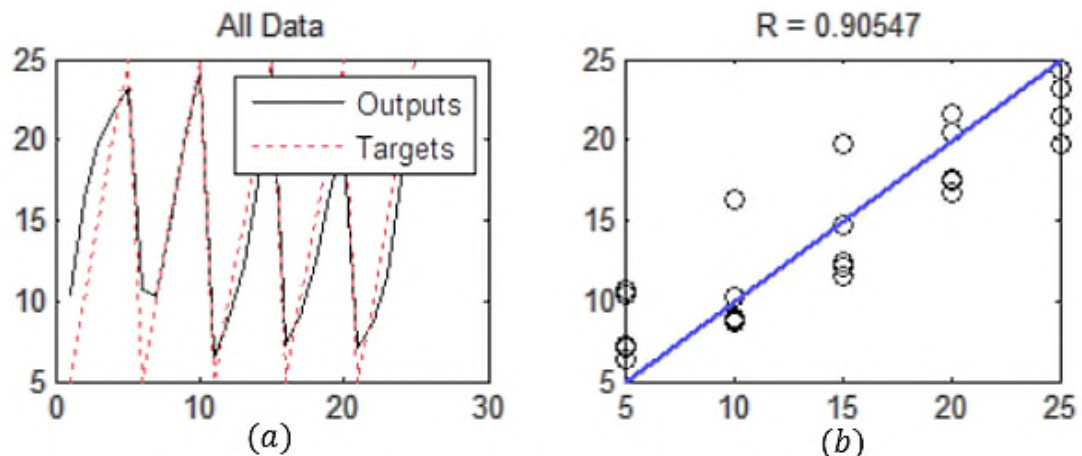

MSE $=9.4457$, RMSE $=3.0734$

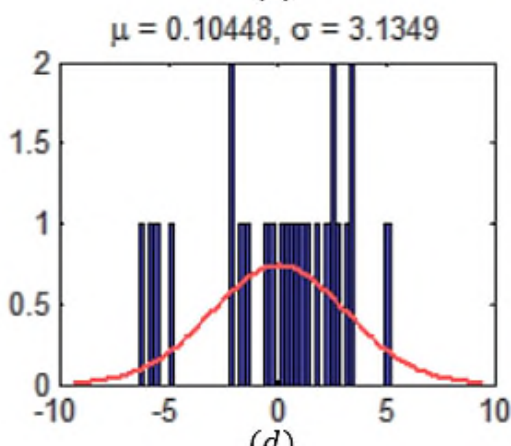

(c)

$(d)$

Fig. 3: The results of neural network (MLP) for all of the slump data 
Bulletin de la Société Royale des Sciences de Liège, Vol. 86, special edition, 2017, p. 855-868

Table 4: The results of neural network (MLP) related to the compressive strength data

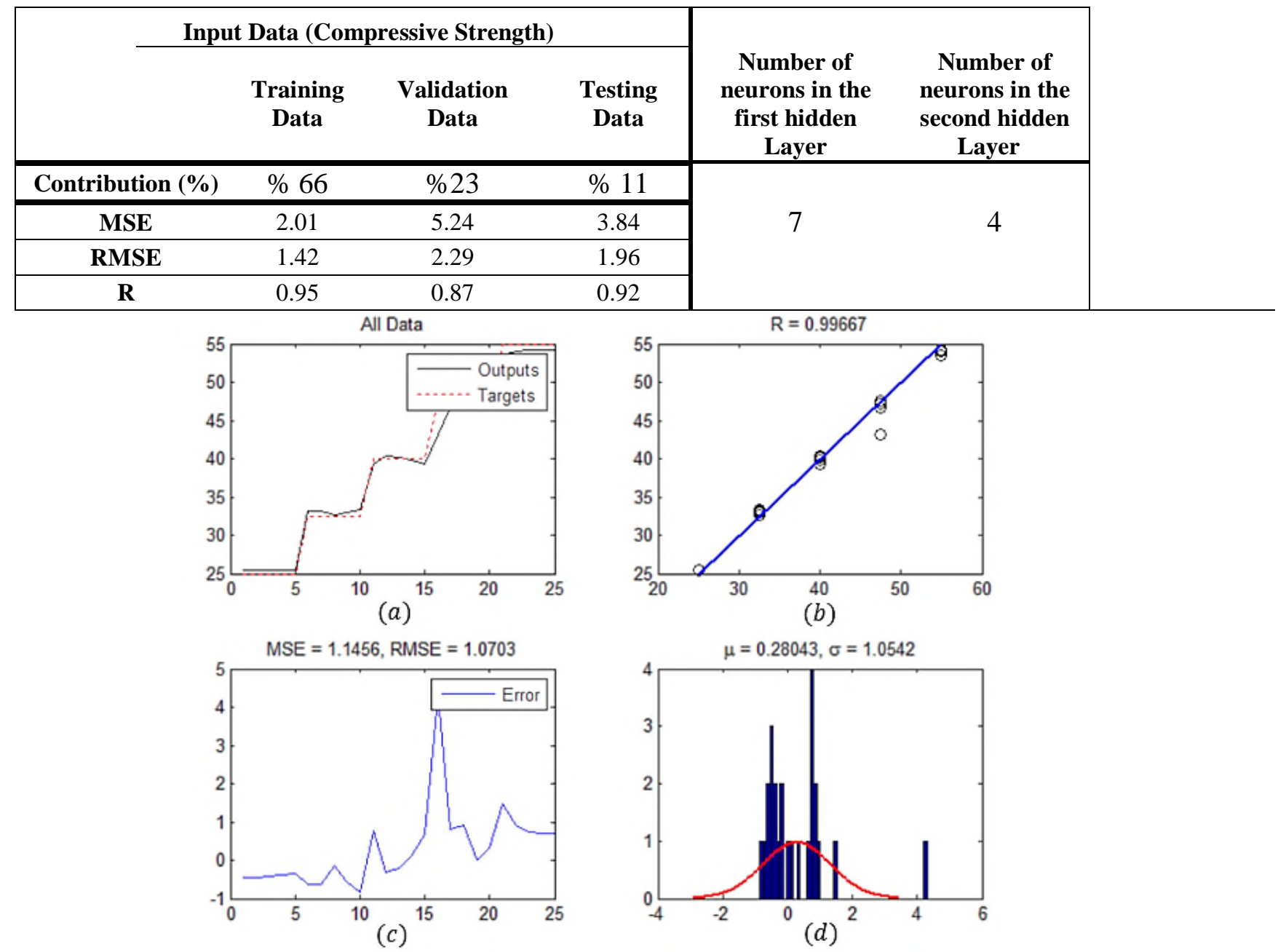

Fig. 4: The results of neural network (MLP) for all of the compressive strength data

The values of standard deviation $(\sigma)$ and root mean square error (RMSE) are almost equal if the mean $(\mu)$ shifts toward zero, neglecting the value of 1 for the bias.

$$
\begin{gathered}
\sigma=\sqrt{\frac{1}{N-1} \sum_{i=1}^{N}\left(e_{i}-\bar{e}\right)^{2}} \\
R M S E=\sqrt{\frac{1}{N} \sum_{i=1}^{N} e_{i}^{2}}
\end{gathered}
$$

The results in GMDH by selecting the threshold criterion $\alpha$ based on Root Mean Square Error (RMSE) were obtained as follows: 
Bulletin de la Société Royale des Sciences de Liège, Vol. 86, special edition, 2017, p. 855-868

Table 5: The results of GMDH neural network associated with the slump data

\begin{tabular}{|c|c|c|c|c|c|}
\hline \multicolumn{3}{|c|}{ Input data (slump) } & \multirow{2}{*}{$\begin{array}{c}\text { Selection } \\
\text { pressure }(\alpha)\end{array}$} & \multirow{2}{*}{$\begin{array}{l}\text { Max Layer- } \\
\text { Neurons }\end{array}$} & \multirow{2}{*}{$\begin{array}{c}\text { Max } \\
\text { Layers }\end{array}$} \\
\hline \multicolumn{2}{|c|}{ Training data } & Test data & & & \\
\hline Contribution (\%) & $\% 70$ & $\% 30$ & \multirow{4}{*}{0.6} & \multirow{4}{*}{50} & \multirow{4}{*}{4} \\
\hline MSE & 3.68 & 5.52 & & & \\
\hline RMSE & 1.92 & 2.35 & & & \\
\hline $\mathbf{R}$ & 0.98 & 0.88 & & & \\
\hline
\end{tabular}

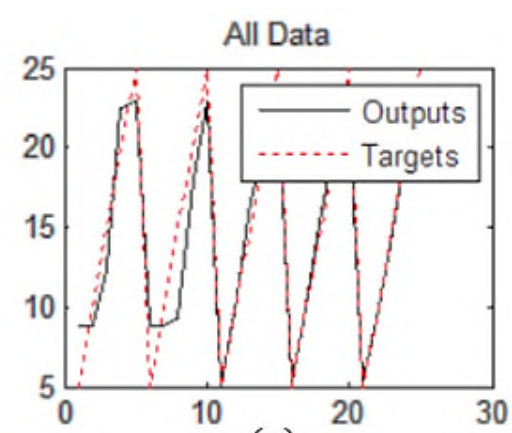

(a)

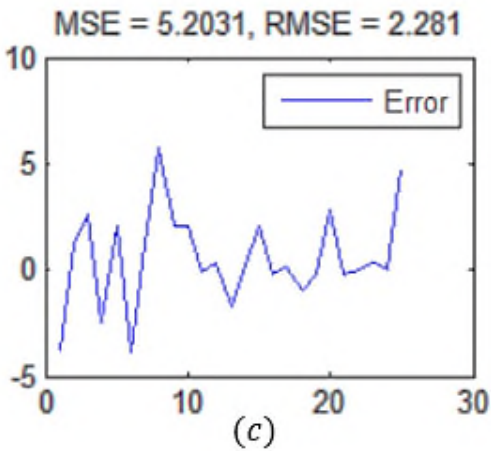

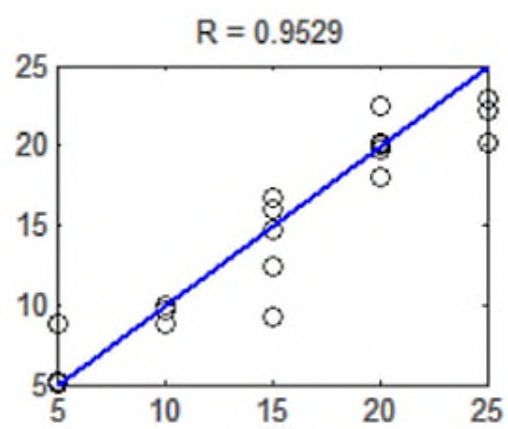

(b)

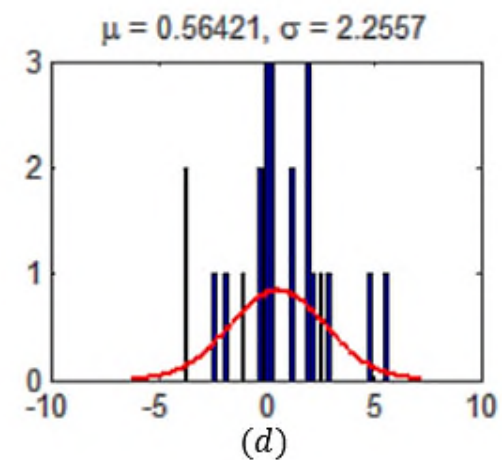

Fig. 5: The results of neural network (GMDH) for all of the slump data 
Bulletin de la Société Royale des Sciences de Liège, Vol. 86, special edition, 2017, p. 855-868

Table 6: The results of neural network (GMDH) associated with the compressive strength data

\begin{tabular}{|c|c|c|c|c|c|}
\hline \multicolumn{3}{|c|}{ Input data (Compressive strength) } & \multirow{2}{*}{$\begin{array}{c}\text { Selection } \\
\text { pressure }(\alpha)\end{array}$} & \multirow{2}{*}{$\begin{array}{c}\text { Max Layer- } \\
\text { Neurons }\end{array}$} & \multirow{2}{*}{$\begin{array}{c}\text { Max } \\
\text { Layers }\end{array}$} \\
\hline \multicolumn{2}{|c|}{ Training data } & Test data & & & \\
\hline Contribution (\%) & $\% 75$ & $\% 25$ & \multirow{4}{*}{0.6} & \multirow{4}{*}{30} & \multirow{4}{*}{5} \\
\hline MSE & 1.98 & 2.75 & & & \\
\hline RMSE & 1.41 & 1.66 & & & \\
\hline $\mathbf{R}$ & 0.96 & 0.91 & & & \\
\hline
\end{tabular}

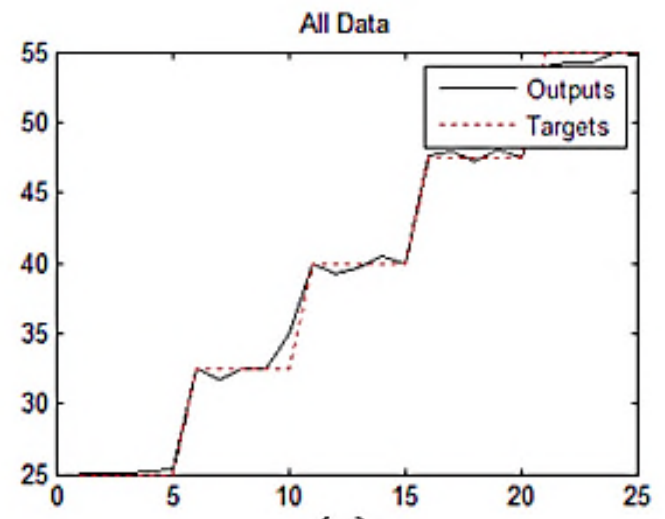

(a)

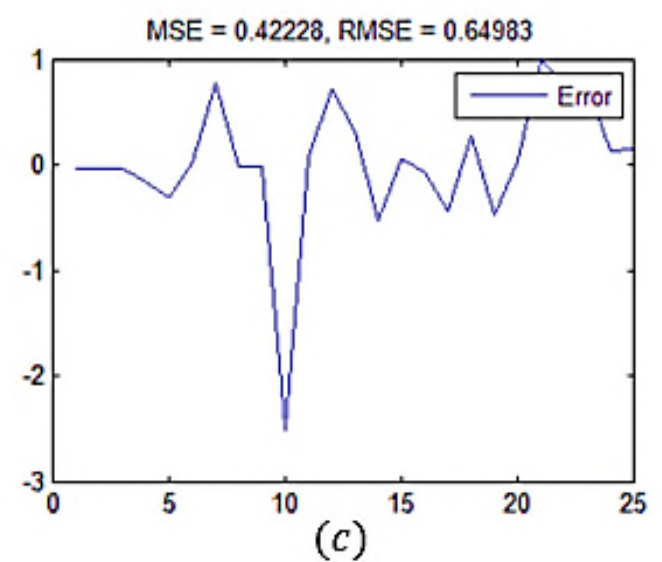

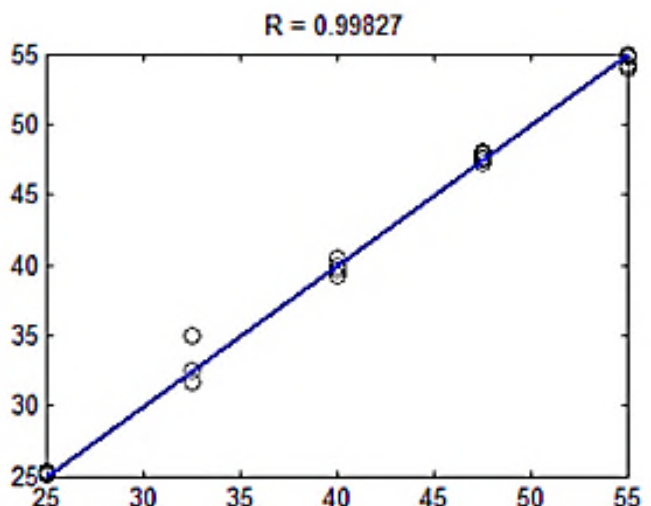

(b)

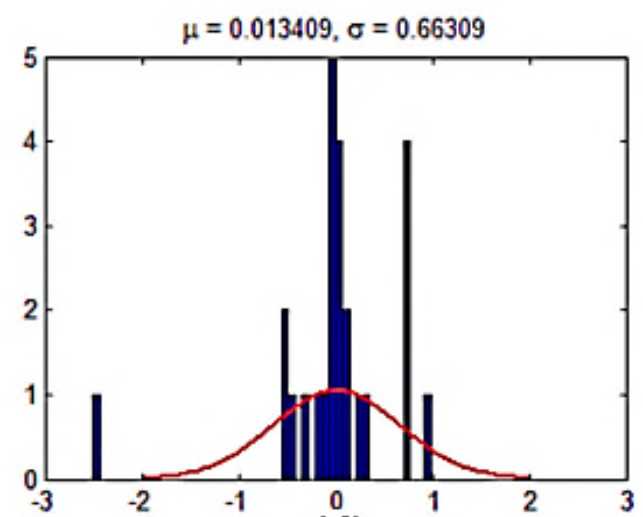

(d)

Fig. 6: The results of neural network (GMDH) for all of the compressive strength data

\section{Conclusion}

The results of modeling for all of the data have been presented in four diagrams for each method (Figs. 3-6), where diagram (a) demonstrates the target values and the values obtained from network for 25 plotted mix designs. Furthermore, Diagram (b) indicates these values with 
correlation factor (R). Diagram (c) manifests the values of error for the 25 mixed designs together with mean square error (MSE) and root mean square error (RMSE) across all of the available data. Finally, diagram (d) reveals the distribution of this error along with the values of standard deviation and mean. The results of conducted calculations suggest the ability of artificial neural networks in the prediction and modeling of nonlinear systems, with the only difference being the fact that the group method of data handling (GMDH) algorithm has been more successful than multilayer perceptron (MLP) neural network in estimating the experimental values of compressive strength and slump. In addition, the RMSE has been lower in GMDH approach for all of the data (1.07 in comparison with 3.07 and 0.64 in comparison with 2.28 for slump values and compressive strength values, respectively).

The experimental results imply the acceptable accuracy of proposed GMDH algorithm. The notable point in this method is the self-organizing characteristic of the network and its high flexibility, making it a powerful instrument for prediction of a variety of nonlinear complex systems. The proposed methodology provides a guideline to model material characteristics using only a limited amount of experimental data. Using this new approach, it is expected that an optimized and efficient algorithm can be estimated for predicting the mechanical properties of concrete containing slag, fly ash, and SP and better results are obtained in comparison with other conventional methods.

\section{Acknowledgement}

This work was conducted in the Construction Material Institute (CMI) at University of Tehran, Iran. The authors would like to acknowledge the support which has made this institute and this research possible.

\section{References}

[1] McCulloch, WS., Pitts, W., (1943), "A logic calculus of the ideas immanent in nervous activity", Bull of Math Biophys, No.5, pp. 115-133.

[2] Haykin, S.,(1994), "Neural network: A comprehensive foundation", MacMillan College Publishing Co, New York.

[3] Welstead, S. T., (1994), "Neural network and fuzzy logic applications in C/C", Wiley, New York.

[4] J.Kasperkiewicz, J. Racz, andA.Dubrawski, (1995), "HPC strength prediction using artificial neural network," Journal of Computing in Civil Engineering, vol. 9, no. 4, pp. 279-284.

[5] Yeh, I-C. , (1998), "Modeling concrete strength with augment-neuron networks." J. Mater. Civ. Eng. 10_4_, 263-268.

[6] Bai,J., (2003), " Using neural networks to predict workability of concrete incorporating metakaolin and fly ash." Advances in Engineering Software 34 663-669.

[7] A. Oztas, M. Pala, E. Ozbay, E. Kanca, N. Caglar, and M. A. Bhatti, (2006), "Predicting the compressive strength and slump of high strength concrete using neural network," Construction and Building Materials, vol. 20, no. 9, pp. 769-775

[8] I.-C. Yeh, (2006), "Exploring concrete slump model using artificial neural networks," Journal of Computing in Civil Engineering, vol. 20, no. 3, pp. 217-221

[9] I.-C. Yeh, (2007), "Modeling slump flow of concrete using second order regressions and artificial neural networks," Cement and Concrete Composites, vol. 29, no. 6, pp. 474-480 
[10] W.-H. Chine, H.-H. Hsu, L. Chen, T.-S. Wang, and C.-H. Chiu, (2010), "Modeling slump of concrete using the artificial neural networks," in Proceedings of the International Conference on Artificial Intelligence and Computational Intelligence (AICI'10), pp. 236-239, Sanya, China, October

[11] A. Bilgil, (2011), "Estimation of slump value and Bingham parameters of fresh concrete mixture composition with artificial neural network modelling," Scientific Research and Essays, vol. 6, no. 8, pp. $1753-1765$

[12] Ramezanianpour, Aliakbar. Pilvar, Amirreza. Sobhani, Jafar (1391). "Estimation of compressive strength of concrete using artificial neural network". The fourth Iranian national annual conference of concrete, Tehran.

[13] Sobhani, Bahman. Esmaeilzadeh Shahri, Efat. (1392). "Prediction of slump values as one of the main parameters of efficiency in concretes with a high strength using artificial neural network modeling". Novel computational and optimization techniques in civil engineering, Islamic Azad University, Saghez Branch, Saghez.

[14] D. Tien Bui, B. T. Pham, Q. P. Nguyen, and N. Hoang, (2016), "Spatial prediction of rainfallinduced shallow landslides using hybrid integration approach of Least-Squares Support Vector Machines and differential evolution optimization: a case study in Central Vietnam, "International Journal of Digital Earth, vol. 9, no. 11, pp. 1077-1097

[15] J.-S. Chou and A.-D. Pham, (2015), "Smart artificial firefly colony algorithm-based support vector regression for enhanced forecasting in civil engineering," Computer-Aided Civil and InfrastructureEngineering,vol.30,no.9,pp.715-732

[16] D.-T.VuandN.-D.Hoang, (2016), "Punching shear capacity estimation of FRP-reinforced concrete slabs using a hybrid machine learning approach," Structure and Infrastructure Engineering, vol. 12, no. 9 , pp. $1153-1161$

[17] Ivakhnenko, A.G., (1976), "The group method of data handling in prediction problems", Soviet Automatic Control of Avtomotika 9. pp. 21-30.

[18] Najafzadeh, M., Barani, G., and Hessami Kermani, M., (2014), "Estimation of Pipeline Scour due to Waves by GMDH.” J. Pipeline Syst. Eng. Pract. DOI:10.1061 / (ASCE) PS.19491204.0000171-.

[19] Najafzadeh, M., Barani, G.A. and Azamathulla, H.M., (2013), "GMDH to predict scour depth around a pier in cohesive soils", Applied Ocean Research 40. pp. 35-41. 